\title{
Aprendiendo en base a proyectos en la asignatura Marketing del Grado en Óptica y Optometría
}

\author{
MARÍA Ángeles ReVILLA CAMACHO \\ Departamento de Administración \\ de Empresas y Marketing \\ Universidad de Sevilla \\ arevilla@us.es \\ D.0.I.: http://dx.doi.org/10.12795/JDU.2018.i01.81 \\ Pp.: 1442-1459
}

\section{Resumen}

La asignatura Marketing, materia optativa del tercer curso del grado en Óptica y Optometría, es la primera y casi única toma de contacto de los estudiantes con la vertiente comercial de su formación. Tras cuatro semestres centrados en la correcta atención al paciente, han de enfrentarse ahora a un cambio de mentalidad, pues deben empezar a ver a los pacientes también como clientes. En este curso académico 2018/2019, al objeto de mejorar la asimilación de conceptos y procedimientos y aumentar la motivación e interés de los estudiantes, se ha realizado un ciclo de mejora basado en un proyecto, de 7 horas de duración. Este ciclo consistía en la selección de la cartera de productos y servicios de un establecimiento de óptica y optometría de nueva creación. Los resultados muestran una elevada implicación, una mejora de la participación y la interacción entre pares, y una adecuada asimilación de los contenidos previstos. 
Palabras clave: Marketing, Grado en Óptica y Optometría, Docencia Universitaria, Experimentación Docente Universitaria, Aprendizaje Basado en Proyectos

\section{Descripción del contexto}

Marketing es una materia optativa del tercer curso del gado en Óptica y Optometría, que escogen de forma regular el $50 \%$ de los estudiantes potenciales, decantándose los restantes por la materia Audiología. Es la primera y única materia en el plan de estudios de dicho grado de un área de conocimiento relacionada con la empresa, por lo que el grado de familiaridad de los estudiantes con la terminología básica es prácticamente nulo. Tampoco tienen conocimientos previos formalizados, puesto que ninguno procede de bachilleratos de Ciencias Sociales. No obstante, en el momento de aplicar el ciclo de mejora llevamos ya suficientes semanas de curso como para que empiecen a interiorizar los principales valores que propone el marketing, e incluso son capaces de efectuar razonamientos coherentes sobre la gestión del mismo en la óptica.

El grupo está integrado por veinticinco estudiantes, pero tres de ellos no asisten a clase con regularidad, por encontrarse trabajando (una de ellas) o estudiando en un curso superior con coincidencia horaria (dos de ellas). El aula es multimedia, con tecnología de pizarra digital, enchufes en las mesas y mobiliario versátil, por lo que es posible modificar la disposición del espacio para trabajar cómodamente en equipo. Dispone también de una pizarra portátil, adquirida recientemente a petición mía. Los medios, por tanto, son adecuados para cualquier iniciativa de innovación docente, y los alumnos son bastantes receptivos a las diferentes propuestas que les he ido haciendo, y participan de forma habitual en las clases. Las sesiones se desarrollan a lo largo de quince semanas, en el primer cuatrimestre del curso, con una duración de cuatro horas semanales, divididas en dos días, martes y jueves, de 12:00-14:00. Es un horario que sólo se aplica a las optativas, ya que el resto de materias acaban y empiezan a las y media, no a las en punto. Ello quiere decir que los 
estudiantes están esperando 30 minutos entre la clase anterior y la mía, lo que afecta a su percepción sobre la duración de la clase (a las 13:30, su reloj biológico les indica que la clase debería terminar).

\section{El aprendizaje basado en proyectos}

Barrows (1986) define al aprendizaje basado en proyectos (ABP) como "un método de aprendizaje basado en el principio de usar problemas como punto de partida para la adquisición e integración de los nuevos conocimientos". El ABP es un modelo de aprendizaje que permite a los estudiantes un trabajo activo, basado en actividades con aplicación al entorno profesional real (Martí et al, 2010). Este modelo guía al individuo en el camino para la obtención de los conceptos, haciéndole responsable de su propio aprendizaje, sin que el docente utilice la lección magistral u otro método para transmitir ese temario. El profesor se convierte en un referente que orienta y guía a los estudiantes y los motiva para alcanzar los resultados previstos (Maldonado, 2008), por lo que su papel como protagonista principal del proceso de enseñanza desaparece. El ABP trabaja formando equipos de estudiantes que deben abordar problemas reales, involucrándolos en proyectos complejos y significativos. Entre sus principales beneficios destacan la mejora en la adquisición de competencias (de Miguel, 2005), el desarrollo del pensamiento crítico y la toma de conciencia del propio aprendizaje (Prieto, 2006), el desarrollo de la creatividad (Benito y Cruz, 2005) y el desarrollo de habilidades de investigación (Servicio de Innovación Educativa UPM, 2008). También mejora la asistencia a clase, el compromiso con la asignatura y los resultados académicos (Revilla y López, 2010). 


\section{Diseño del Ciclo de Mejora}

Este ciclo de mejora se inicia en la octava semana del curso, con los contenidos correspondientes al tema 3 de la asignatura, centrados en la estrategia de producto, servicio y marca. Con respecto a la planificación del ciclo de mejora, comenzó por el diseño del mapa de contenidos (figura 1), siguiendo las recomendaciones de Porlán et al (2017).

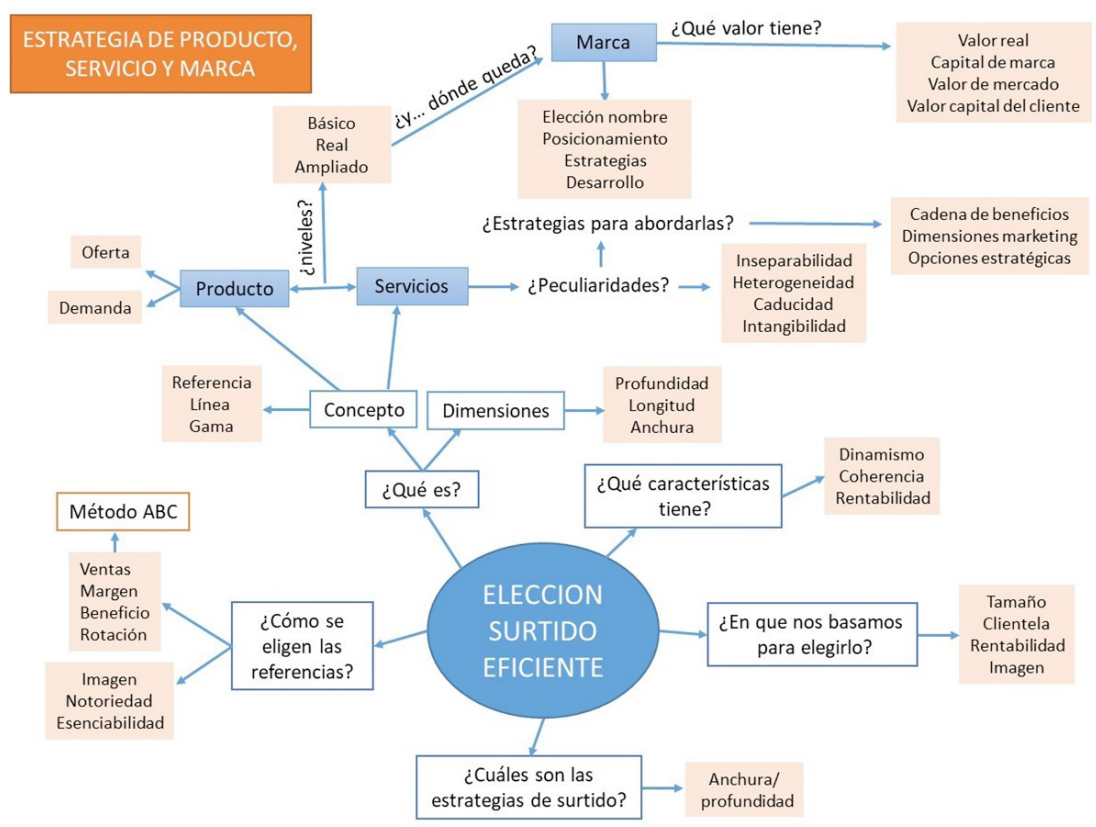

Figura 1. Mapa de contenidos empleado en el ciclo de mejora.

El mapa parte de un concepto central: el surtido eficiente, que implica la elección de los productos y servicios que un establecimiento debe vender para satisfacer al cliente y ser rentable. En torno a él se definen las cualidades que debería tener un surtido para ser eficiente, las cuestiones que debemos tener en cuenta para determinar ese surtido, y las posibles estrategias comerciales. Todos estos son contenidos conceptuales, a los que sigue un contenido procedimental, como es la aplicación del método $A B C$ de selección de referencias. Teniendo en cuenta que se trabajará este procedimiento facilitando información 
real a los estudiantes, el ciclo incidirá también en la capacidad de seleccionar la información relevante de entre un conjunto de datos, contenido de tipo procedimental. Con respecto a las actividades que se plantean, implicarán el desarrollo de competencias actitudinales, ya que se busca el desarrollo de una actitud de tolerancia al cambio, el fomento del espíritu crítico y la escucha activa.

La primera y la última actividad del ciclo consiste en la cumplimentación del siguiente cuestionario, diseñado para conocer los modelos mentales de los estudiantes y ayudarles a tomar conciencia de su punto de partida.

1. ¿Qué significado tienen las palabras cartera, gama, línea y referencia, cuando estamos diseñando una estrategia de producto?

2. ¿Todas las ópticas tienen que vender de todo o es posible que cada una de ellas elija qué quiere ofrecer en su establecimiento? ¿Por qué?

3. ¿Qué es un surtido eficiente?

4. ¿Cómo se decide qué productos y servicios ofrecer en un establecimiento de óptica?

El modelo metodológico previsto está diseñado con el objetivo de que el docente hable lo menos posible, para dejarles construir su propio aprendizaje sobre este tema, siguiendo las propuestas de Finkel (2008). Por ello, se basará en una actividad grupal. Cada grupo ha de elegir el surtido eficiente de una óptica independiente de nueva creación. Tienen libertad absoluta de actuación, ya que un inversor privado sin conocimientos del sector, les ha ofrecido la financiación necesaria. Sólo les ha exigido transparencia en la gestión, y desarrollo de los principios básicos de la planificación de marketing.

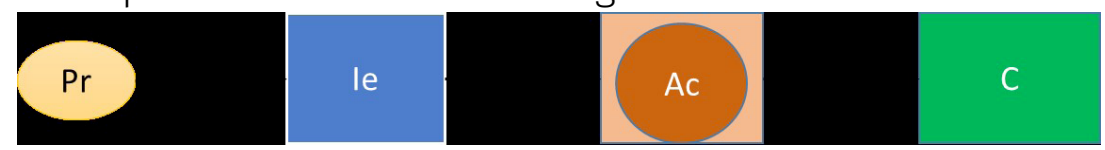

Figura 2. Modelo metodológico previsto

Pr: Presentación del problema y de las instrucciones necesarias para abordarlo 
le: Tiempo de trabajo individual y grupal para que tomen conciencia de la situación inicial y soliciten la información que les haga falta, en su caso, para abordarlo

Ac: Puesta en común de las cuestiones clave que cada grupo ha encontrado

C: Conclusiones finales sobre las pautas generales que se deben aplicar para resolver este tipo de cuestiones.

La secuencia prevista de actividades, a implantar en tres sesiones de clase, comprendidas entre los días 6 y 13 de noviembre, se presenta en la tabla 1.

\section{Tabla 1}

Secuencia de actividades

\begin{tabular}{|c|c|c|c|}
\hline \multicolumn{4}{|c|}{ SESIÓN 1: MARTES, 06 DE NOVIEMBRE } \\
\hline Actividades & Finalidad & Tiempo & Contenidos \\
\hline $\begin{array}{l}\text { Saludo y preparación de } \\
\text { equipos }\end{array}$ & $\begin{array}{l}\text { Romper el hielo y pre- } \\
\text { pararse para abordar el } \\
\text { proceso }\end{array}$ & $\begin{array}{c}5 \\
\text { minutos }\end{array}$ & \multirow{9}{*}{ 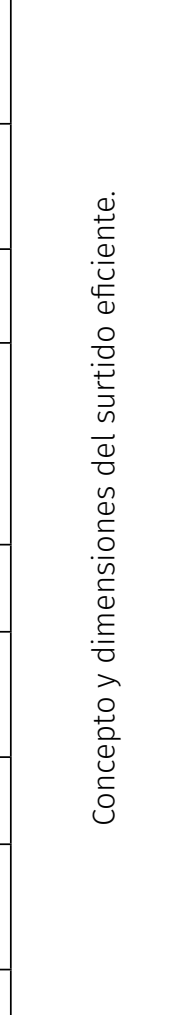 } \\
\hline $\begin{array}{l}\text { Presentación del proyecto } \\
\text { de innovación docente y so- } \\
\text { licitud de colaboración }\end{array}$ & $\begin{array}{l}\text { Despertar una actitud po- } \\
\text { sitiva y reducir la ansiedad }\end{array}$ & 5 minutos & \\
\hline $\begin{array}{l}\text { Entrega y cumplimentación } \\
\text { del cuestionario inicial }\end{array}$ & $\begin{array}{l}\text { Conocer los modelos men- } \\
\text { tales previos }\end{array}$ & 20 minutos & \\
\hline $\begin{array}{l}\text { Planteamiento y contextuali- } \\
\text { zación del problema: deben } \\
\text { abrir una óptica y hay que } \\
\text { decidir los productos y ser- } \\
\text { vicios que va a comercializar } \\
\end{array}$ & $\begin{array}{l}\text { Captar la atención de los } \\
\text { estudiantes y generar inte- } \\
\text { rés por la actividad }\end{array}$ & $\begin{array}{c}5 \\
\text { minutos }\end{array}$ & \\
\hline $\begin{array}{l}\text { Entrega de documentación } \\
\text { necesaria }\end{array}$ & $\begin{array}{l}\text { Transmitir conocimiento } \\
\text { sobre los contenidos }\end{array}$ & 15 minutos & \\
\hline $\begin{array}{l}\text { Constitución de equipos y } \\
\text { entrega de guión con las de- } \\
\text { cisiones a tomar }\end{array}$ & $\begin{array}{l}\text { Orientar el trabajo, presen- } \\
\text { tando las preguntas a las } \\
\text { que deben dar respuesta }\end{array}$ & 10 minutos & \\
\hline $\begin{array}{l}\text { Trabajo individual de lectura } \\
\text { detenida del guión }\end{array}$ & $\begin{array}{l}\text { Comprensión profunda del } \\
\text { problema a abordar }\end{array}$ & 15 minutos & \\
\hline $\begin{array}{l}\text { Trabajo grupal autónomo. } \\
\text { Guía de ese proceso a de- } \\
\text { manda de cada equipo. }\end{array}$ & $\begin{array}{l}\text { Fomentar el razonamiento, } \\
\text { la discusión y la búsqueda } \\
\text { de información relevante } \\
\end{array}$ & 40 minutos & \\
\hline Despedida & & 5 minutos & \\
\hline
\end{tabular}

Jornadas de Formación e Innovación Docente del Profesorado | № 1 (2018) Esta obra se distribuye con la licencia Creative Commons Reconocimiento-NoComercial-SinObraDerivada Internacional (CC BY-NC-ND 4.0.) 
SESIÓN 2: JUEVES, 08 DE NOVIEMBRE

\begin{tabular}{|c|c|c|c|}
\hline Actividades & Finalidad & Tiempo & Contenidos \\
\hline $\begin{array}{l}\text { Saludo y preparación de } \\
\text { equipos }\end{array}$ & $\begin{array}{l}\text { Romper el hielo y pre- } \\
\text { pararse para abordar el } \\
\text { proceso }\end{array}$ & $\begin{array}{c}5 \\
\text { minutos }\end{array}$ & \multirow{7}{*}{ 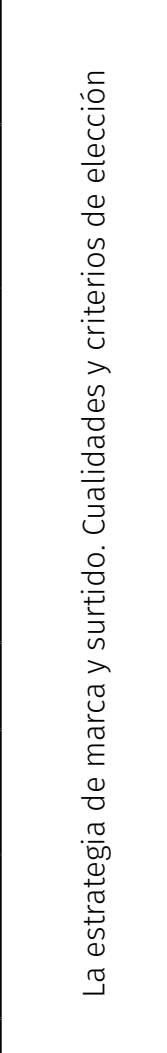 } \\
\hline $\begin{array}{l}\text { Exposición por parte del } \\
\text { profesor de las principales } \\
\text { cuestiones abordadas en la } \\
\text { sesión anterior }\end{array}$ & $\begin{array}{l}\text { Captar la atención y reto- } \\
\text { mar la actitud y tensión de } \\
\text { la sesión anterior }\end{array}$ & $\begin{array}{c}5 \\
\text { minutos }\end{array}$ & \\
\hline $\begin{array}{l}\text { Exposición de cada grupo, } \\
\text { respondiendo a las cues- } \\
\text { tiones clave: ¿Qué tipos de } \\
\text { productos y servicios voy a } \\
\text { incluir en mi cartera? ¿Qué } \\
\text { estrategias voy a desarrollar } \\
\text { para salvar la problemática } \\
\text { que conlleva ser, en esencia, } \\
\text { una empresa de servicios? }\end{array}$ & $\begin{array}{l}\text { Desarrollar habilidades de } \\
\text { presentación oral, asi como } \\
\text { hacer una crítica cons- } \\
\text { tructiva de las soluciones } \\
\text { propuestas, que guíe el } \\
\text { aprendizaje }\end{array}$ & 20 minutos & \\
\hline $\begin{array}{l}\text { Resumen de conclusiones } \\
\text { sobre la materia abordada }\end{array}$ & $\begin{array}{l}\text { Asentamiento de los con- } \\
\text { ceptos trabajados }\end{array}$ & 10 minutos & \\
\hline $\begin{array}{l}\text { Trabajo en grupo autónoma. } \\
\text { Guía de ese proceso a de- } \\
\text { manda de cada equipo. }\end{array}$ & $\begin{array}{l}\text { Fomentar el razona- } \\
\text { miento, la discusión y la } \\
\text { argumentación }\end{array}$ & 60 minutos & \\
\hline $\begin{array}{l}\text { Respuesta a la cuestión: } \\
\text { ¿Voy a crear un nombre co- } \\
\text { mercial, una marca, para mi } \\
\text { establecimiento? }\end{array}$ & $\begin{array}{l}\text { Asentamiento de los con- } \\
\text { ceptos trabajados }\end{array}$ & 15 minutos & \\
\hline Despedida & & 5 minutos & \\
\hline
\end{tabular}


SESIÓN 3: MARTES, 13 DE NOVIEMBRE

\begin{tabular}{|c|c|c|c|}
\hline Actividades & Finalidad & Tiempo & Contenidos \\
\hline $\begin{array}{l}\text { Saludo y preparación de } \\
\text { equipos }\end{array}$ & $\begin{array}{l}\text { Romper el hielo y pre- } \\
\text { pararse para abordar el } \\
\text { proceso }\end{array}$ & 5 minutos & \multirow{9}{*}{ 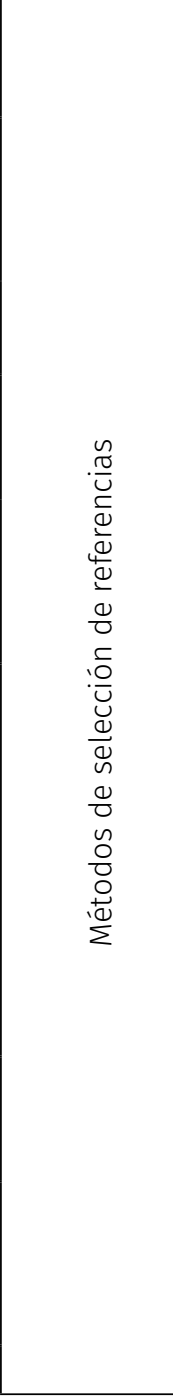 } \\
\hline $\begin{array}{l}\text { Exposición por parte del } \\
\text { profesor de las principales } \\
\text { cuestiones abordadas en la } \\
\text { sesión anterior }\end{array}$ & $\begin{array}{l}\text { Captar la atención y reto- } \\
\text { mar la actitud y tensión de } \\
\text { la sesión anterior }\end{array}$ & 5 minutos & \\
\hline $\begin{array}{l}\text { Explicación profesor mé- } \\
\text { todo } A B C\end{array}$ & $\begin{array}{l}\text { Dotar de herramientas } \\
\text { para resolver caso }\end{array}$ & 15 minutos & \\
\hline $\begin{array}{l}\text { Trabajo individual de lec- } \\
\text { tura detenida de la informa- } \\
\text { ción presentada }\end{array}$ & $\begin{array}{l}\text { Comprensión profunda del } \\
\text { problema a abordar }\end{array}$ & 10 minutos & \\
\hline $\begin{array}{l}\text { Trabajo en grupo autónomo. } \\
\text { Guía de ese proceso a de- } \\
\text { manda de cada equipo. }\end{array}$ & $\begin{array}{l}\text { Fomentar el razonamiento, } \\
\text { la discusión y la argumen- } \\
\text { tación, y la búsqueda de } \\
\text { información relevante }\end{array}$ & 35 minutos & \\
\hline $\begin{array}{l}\text { Presentación de resultados } \\
\text { de cada grupo: ¿Qué dimen- } \\
\text { siones vamos a darle al sur- } \\
\text { tido, es decir, cuál va a ser } \\
\text { la estrategia comercial en } \\
\text { relación al surtido? ¿Qué } \\
\text { métodos voy a elegir para } \\
\text { asegurar que mi surtido } \\
\text { tiene las cualidades básicas } \\
\text { de un surtido eficiente? }\end{array}$ & $\begin{array}{l}\text { Realizar un contraste } \\
\text { para conocer las distintas } \\
\text { posturas }\end{array}$ & 10 minutos & \\
\hline $\begin{array}{l}\text { Presentación solución co- } \\
\text { rrecta o más adecuada }\end{array}$ & $\begin{array}{l}\text { Conclusión final del pro- } \\
\text { fesor que fije las ideas } \\
\text { principales }\end{array}$ & 15 minutos & \\
\hline $\begin{array}{l}\text { Entrega y cumplimentación } \\
\text { del cuestionario final }\end{array}$ & $\begin{array}{l}\text { Indagar sobre los resulta- } \\
\text { dos del proceso de apren- } \\
\text { dizaje, sobre la evolución } \\
\text { de cada estudiante }\end{array}$ & 20 minutos & \\
\hline Despedida y agradecimiento & & 5 minutos & \\
\hline
\end{tabular}

Jornadas de Formación e Innovación Docente del Profesorado | № 1 (2018) Esta obra se distribuye con la licencia Creative Commons Reconocimiento-NoComercial-SinObraDerivada Internacional (CC BY-NC-ND 4.0.) 


\section{Aplicación del Ciclo de Mejora}

El día 6 de noviembre de 2018 comienza el ciclo de mejora de la asignatura, con 22 estudiantes presentes en el aula. Para ponerlos en situación, les he explicado que estamos participando en un curso de innovación docente, y que agradezco su colaboración en el desarrollo de este ciclo de mejora. También les he asegurado que el esfuerzo adicional que harán, en su caso, será objeto de evaluación, siempre de forma positiva.

Tras esta pequeña introducción, les hago entrega del cuestionario inicial. La mayoría se asusta, piensa que es un examen, y se pone a la defensiva, porque yo no he explicado nada y ellos no tienen ni idea de cómo responder. Les tranquilizo, explicándoles por qué hacemos el cuestionario, y el valor que tiene para el diseño del ciclo. También les cuento que ese mismo cuestionario lo he testado con mis hijos adolescentes, que no saben nada sobre el tema, y que han contestado a todas las preguntas. Además, que recuerden que estamos en un proyecto de innovación y que es su oportunidad de echar su imaginación a volar y tratar de responder a las cuestiones según su criterio. Y, lo que es más importante, que no les voy a poner nota. Tras un breve, pero sobrecogedor silencio, todos se ponen a escribir y se concentran en la tarea de responder a las cuestiones. Pasado el tiempo previsto, recojo los cuestionarios, les doy las gracias y paso a explicarles la secuencia prevista de la sesión.

En este punto, incido en la idea de que no voy a explicar nada de forma transmisiva $y$, de hecho, les hago ver que no he recogido en conserjería las llaves, el pen, el mando y todo lo necesario para usar los equipos multimedia. Serán tres días sin power-point, y yo misma me he comprometido a ello, al no traer ningún apoyo para la clase, ni siquiera una simple tiza.

También es preciso aclarar que, aunque las actividades están secuenciadas para las tres sesiones, realmente 
integran un único proyecto, por lo que decido entregarles el guión completo de las tres sesiones, el mapa de contenidos impreso, un ejemplar de la presentación del tema que está colgada en la plataforma de enseñanza virtual, y una copia del BOJA donde se regula el funcionamiento de los establecimientos de óptica en Andalucía. Creo que esto permitirá que cada grupo vaya avanzando al ritmo que necesite, lo que considero vital en el proceso de enseñanza basado en proyectos. El documento recoge las preguntas a las que deben responder, cinco grandes cuestiones integradas cada una de ellas por tres actividades secuenciales, encaminadas a seleccionar el surtido de un establecimiento óptico de nueva creación.

Una vez entregado el documento completo, los animo a colocarse por equipos y empezar a trabajar. Yo me bajo de la tarima y me pongo cerca de ellos. Están agobiados, se les nota. Piensan que es una tarea imposible de abordar, que me he vuelto loca y que no serán capaces de hacerla. No saben por dónde empezar, así que vuelvo a intervenir, les digo que yo empezaría leyendo la tarea, y descubriendo qué hay que hacer. Que, tras ello, procuren ir paso a paso, buscando en la información que les he suministrado los datos necesarios para ello. Que debatan en el grupo la utilidad de la información y el significado de la misma. Y que acudan a mí cuando lo estimen necesario, pero sólo dos veces por equipo, por aquello de dar oportunidades a todos de contar con mi asesoramiento. Aprovecho para decirles que creo en ellos, que son capaces, que me han demostrado a lo largo del curso que son un grupo de estudiantes proactivos y con iniciativa. Y que se trata de montar SU negocio, con libertad total para ello. Tras esto, que parece surtir efecto, se ponen a trabajar, primero en silencio total y más adelante empezando a debatir entre ellos. Acudo a la llamada de todos los equipos un par de veces y sin darnos cuenta, por primera vez en todo el curso, llegan las 14:00 y damos la clase por concluida. 
La mayoría ha llegado a responder a dos puntos de la primera cuestión.

El jueves 08 de noviembre tenemos la segunda sesión del ciclo de mejora, que comienza de una forma sorprendente, al menos para mí. Cuando llego al aula, me encuentro a los alumnos ya sentados por grupos y trabajando de forma autónoma. Tras saludarlos, procedo a hacer un pequeño resumen del punto de partida, pidiéndoles a cada uno de los equipos que nos cuente por dónde van y qué han definido de la tarea. La mayoría está en el punto esperado, pero hay un grupo más adelantado, ya por la segunda cuestión, y otro que aún está en los inicios de la tarea. Una vez comprobado en qué punto está cada equipo, y poner de manifiesto los conceptos del mapa de contenidos que ya hemos trabajado, paso a atender las demandas de los equipos, algo en lo que transcurre toda la clase. Durante ese tiempo, me doy cuenta de que repito siete veces las mismas cuestiones teóricas y procedimentales, pero aplicadas a cada una de las ópticas teóricas definidas por los estudiantes. Soy una consultora en toda regla, a la que plantean cuestiones que, más que responder, aprovecho para convertir en actividades de contraste, que les hagan ver aspectos del problema que no habían contemplado y, en base a ellos, responder ellos mismos a las cuestiones. De nuevo agotamos los 120 minutos, sin que nadie lo haya puesto de manifiesto y, lo que es más llamativo, sin que me hayan pedido el parte de firmas, que paso a diario para controlar la asistencia a clase, con un peso del 10\% sobre la calificación final de la asignatura. La mayoría de equipos están cumpliendo la planificación prevista, a pesar de que ellos no habían sido informados de qué preguntas debían abordar en cada sesión. El ritmo planificado, por tanto, parece adecuado a las tareas asignadas.

Llega el día en que está planificado acabar el ciclo de mejora, 13 de noviembre. De nuevo me sorprenden, están trabajando. Pero los noto cansados, parece que la fase que abordan ahora, la última ya, les pesa. Por ese motivo, decido dinamizar la sesión estableciendo un rato de exposición colectiva de las cuestiones que necesitan respuesta. No utilizo el método transmisivo, simplemente les pongo 
ejemplos de empresas del sector, y de otros sectores, y les pregunto por aspectos de sus estrategias relacionados con la temática que tienen que abordar en esta sesión. El debate los despierta, y pasan de nuevo a trabajar con más motivación. Les doy una sesión más de consultoría a cada grupo, aprovechando para resaltar los puntos fuertes de sus propuestas y ayudarles a encontrar las debilidades. Todos están ya en el último punto, las cuestiones referidas a los métodos de determinación del surtido eficiente. Es en este punto donde decido explicar brevemente cómo se debe proceder al cálculo numérico de las referencias (método $A B C$ ) y les pido que lo trabajen de forma individual para la siguiente sesión.

En ella, ya el día 15 de noviembre, resolvemos el caso numérico, que es común para todos, y hacemos una breve exposición de cada una de las estrategias de producto y marca que han desarrollado. Aprovecho para afianzar conceptos, siempre a partir de las cuestiones que plantean y de las estrategias que exponen. Y les paso el cuestionario final, que acogen con confianza e interés, Todo ello nos supone unos 50 minutos de tiempo, a los que yo añado otros 10 preguntándoles qué les ha parecido la experiencia, si consideran que es un método eficaz y motivador. Aunque hay de todo, la mayoría cree que saben más de este tema que del resto que hemos trabajado, no tanto en lo referente a los conceptos, sino en lo relativo a los procedimientos. Piensan que han aprendido a tomar decisiones sobre la cartera de productos de la óptica, y que es un conocimiento de utilidad para su futuro. Pero han tenido que esforzarse mucho, según me comentan, y no saben si ese esfuerzo se notará en la prueba de evaluación de la asignatura (examen tradicional). Por mi parte, estoy muy satisfecha con su trabajo e implicación, y así se lo hago saber. Les doy las gracias por su actitud y su capacidad de trabajar de forma autónoma, y les aseguro que estoy convencida que su esfuerzo y el grado de conocimientos que han adquirido se reflejará en la prueba de evaluación. Este convencimiento se refuerza con el análisis de las escaleras de aprendizaje, que en todos los casos han evidenciado un progreso importante en el aprendizaje (ver la tabla 2). 
Tabla 2. Evolución por estudiante

\begin{tabular}{|c|c|c|c|c|c|c|c|c|c|c|}
\hline & \multicolumn{2}{|c|}{ CUESTIÓN 1 } & \multicolumn{2}{c|}{ CUESTIÓN 2 } & \multicolumn{2}{c|}{ CUESTIÓN 3 } & \multicolumn{2}{c|}{ CUESTIÓN 4 } & \multirow{2}{*}{ ESCALONES } \\
\hline & Inicial & Final & Inicial & Final & Inicial & Final & Inicial & Final & \\
\hline $\mathbf{1}$ & C & B & B & B & B & B & C & B & 2 \\
\hline $\mathbf{2}$ & B & B & C & B & B & B & C & B & 2 \\
\hline $\mathbf{3}$ & B & B & A & A & C & C & C & B & 1 \\
\hline $\mathbf{4}$ & B & B & C & B & B & A & C & B & 3 \\
\hline $\mathbf{5}$ & D & C & B & B & C & A & C & A & 5 \\
\hline $\mathbf{6}$ & C & B & C & B & C & B & C & A & 5 \\
\hline $\mathbf{7}$ & A & A & B & B & C & B & C & A & 3 \\
\hline $\mathbf{8}$ & B & B & B & B & C & B & C & A & 3 \\
\hline $\mathbf{9}$ & C & C & C & B & D & B & C & C & 3 \\
\hline $\mathbf{1 0}$ & C & C & C & B & C & C & B & B & 1 \\
\hline $\mathbf{1 1}$ & B & B & C & B & C & B & C & C & 2 \\
\hline $\mathbf{1 2}$ & B & A & C & B & B & B & C & B & 3 \\
\hline $\mathbf{1 3}$ & C & B & C & B & C & C & C & A & 4 \\
\hline $\mathbf{1 4}$ & C & C & C & B & B & B & C & A & 3 \\
\hline $\mathbf{1 5}$ & C & B & C & B & A & A & B & C & 3 \\
\hline $\mathbf{1 6}$ & D & C & C & B & B & B & C & C & 2 \\
\hline $\mathbf{1 7}$ & A & A & C & B & C & B & C & C & 2 \\
\hline $\mathbf{1 8}$ & A & A & D & C & B & A & C & C & 2 \\
\hline $\mathbf{1 9}$ & B & A & C & C & B & A & C & C & 2 \\
\hline $\mathbf{2 0}$ & C & B & C & C & B & A & C & C & 2 \\
\hline $\mathbf{2 1}$ & B & B & C & C & D & B & C & C & 2 \\
\hline $\mathbf{2 2}$ & D & D & C & A & B & A & C & A & 5 \\
\hline
\end{tabular}

Recogemos a continuación las escaleras de aprendizaje para cada una de las cuestiones planteadas en el cuestionario, tanto inicial como final. Se ha optado por valorar todas las preguntas en base a la misma escala, de acuerdo a los siguientes niveles:

NIVEL A: Contesta de forma razonada y coherente y contempla todos los aspectos relevantes implicados

NIVEL B: Contesta de forma adecuada, y ve varios de los aspectos implicados.

NIVEL C: Contesta de forma coherente, pero sólo contempla un aspecto.

NIVEL D: No contesta adecuadamente, no sabe nada sobre el tema. 


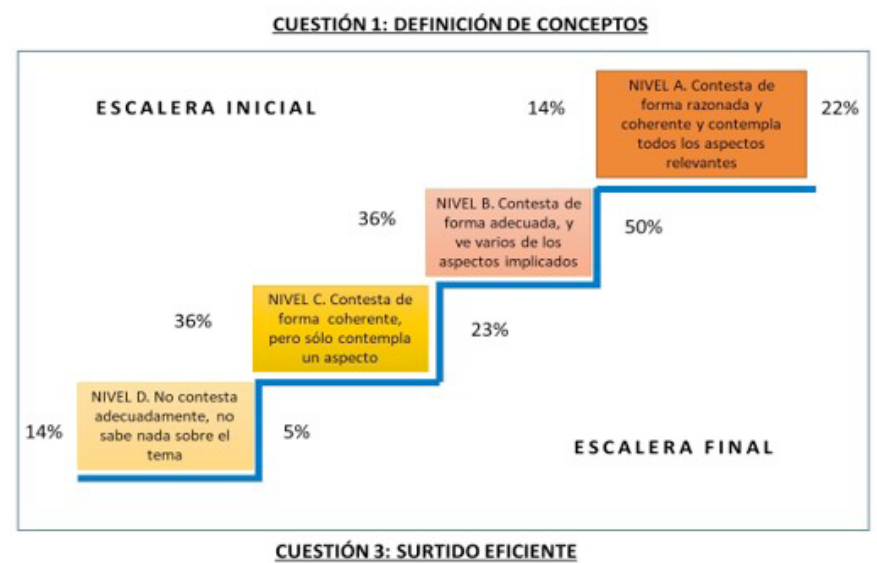

CUESTIÓN 2: ¿TODAS LAS OPTICAS TIENEN QUE VENDER LO MISMO?
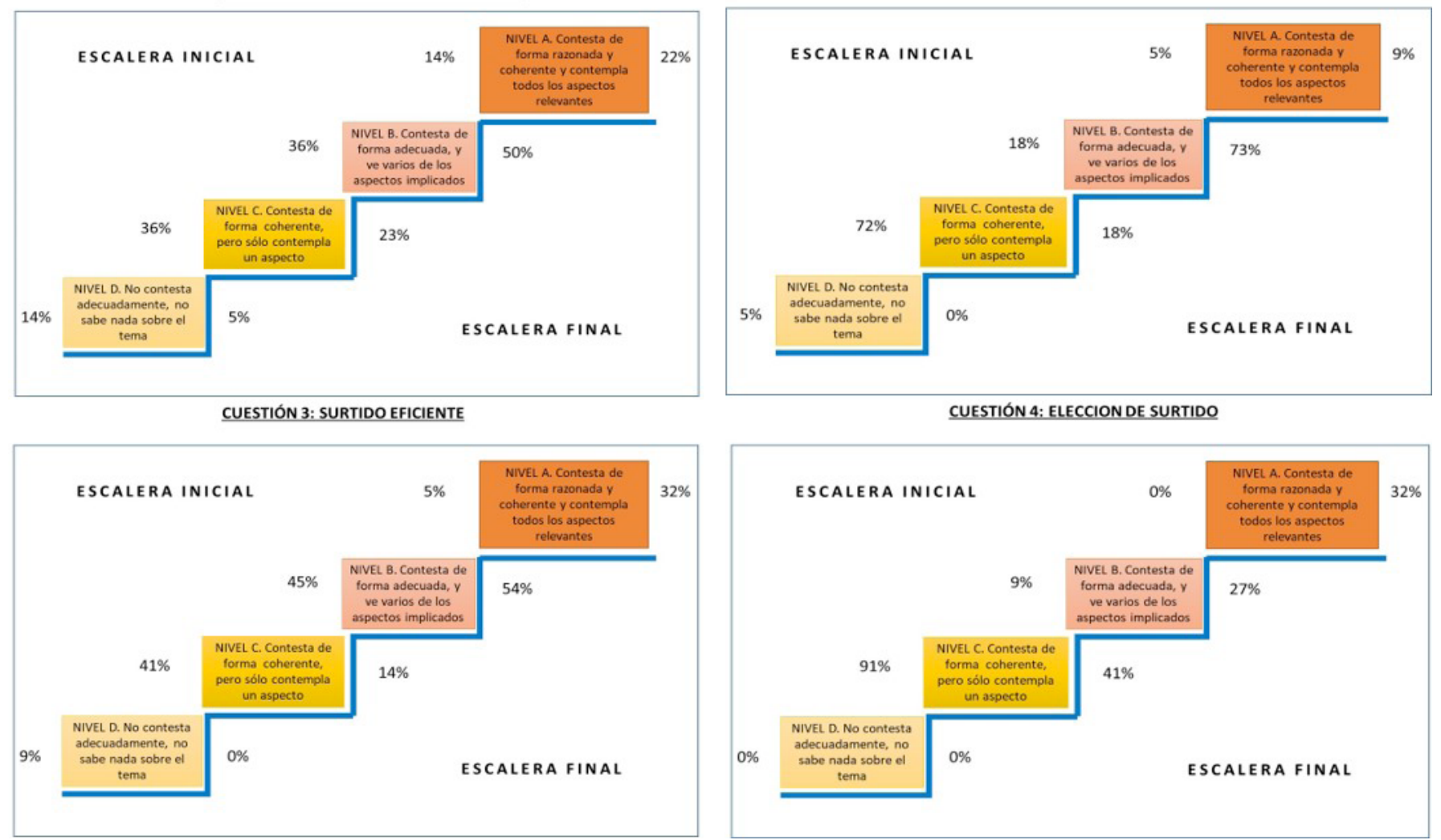

Figura 3. Escaleras de aprendizaje

Jornadas de Formación e Innovación Docente del Profesorado | № 1 (2018)

(c) E) Esta obra se distribuye con la licencia Creative Commons

Internacional (CC BY-NC-ND 4.0.) 
Tras el análisis de los cuestionarios, he podido comprobar que los alumnos tienen ideas preconcebidas sobre materias de las que, en principio, nunca han recibido formación reglada. Esas ideas no suelen acercarse a la realidad de los conceptos, pero es curioso comprobar cómo los errores suelen ser los mismos en diferentes estudiantes, salvo excepciones. Probablemente eso sea debido a su forma de pensar, a sus modelos mentales. Conocerlos se me antoja de una gran importancia para diseñar actividades adecuadas, sobre todo las que les llevan a dar los primeros pasos y los ayudan o guían para ir adoptando un modelo mental más acorde al que deben aplicar para aprender las cuestiones que aborda la materia de aprendizaje.

\section{Evaluación del Ciclo de Mejora}

El modelo didáctico personal por el que yo apuesto es aquél que me permite trabajar durante todo el curso en base a proyectos, planteando un problema complejo y dividiendo ese problema en cuestiones más sencillas, que se constituyan en etapas de aprendizaje y, al mismo tiempo, ayuden a los estudiantes a resolver ese problema principal de forma secuencial. Este modelo exige de un compromiso fuerte del alumnado, que debe olvidar los sistemas tradicionales de docencia, en los que asume un papel pasivo, y apostar decididamente por ser parte activa de su aprendizaje. En este sentido, es importante que los estudiantes tomen conciencia de sus limitaciones en cuanto a la materia de aprendizaje, de la utilidad de la misma para su formación, y de la necesidad de adquirir una serie de competencias y habilidades que dificilmente se pueden aprender simplemente escuchando al docente. La interacción con el alumnado, y de los propios alumnos entre sí, es un factor crítico para poder aplicar este modelo didáctico en la práctica diaria. Por tanto, como primer elemento crítico del cambio de modelo, señalaría a los propios estudiantes, 
que deben cooperar para que el sistema se pueda implantar de forma exitosa.

Para poder aplicarlo con éxito, también es imperativo diseñar actividades motivadoras, útiles y factibles, que fomenten el razonamiento, la discusión y el análisis crítico-reflexivo, y permitan adquirir, de forma natural, las habilidades requeridas. Estas actividades deben basarse en preguntas clave, relacionadas con los conceptos a adquirir y que supongan un reto para los estudiantes. La labor del docente es el segundo elemento clave de esta metodología: hay que reestructurar los contenidos, su tipología y secuencia. Los mapas conceptuales, que muestran cuáles son los contenidos clave y sus relaciones, son una gran ayuda en esta tarea, y es curioso comprobar cómo algunos conceptos y contenidos que nos parecían vitales y que siempre incluíamos en nuestros programas, dejan de serlo, al carecer de encaje en los mapas. En este sentido, he aprendido que aquellos conceptos que no son fáciles de representar en un mapa conceptual, y que no se relacionan con otros, probablemente no sean relevantes y puedan ser suprimidos. No obstante, en materias coordinadas de forma muy centralizada, con profesores que no creen o no tienen tiempo o interés en renovaciones metodológicas, esta supresión de contenidos se antoja complicada.

En cuanto a la evaluación, es el aspecto más complejo de abordar. Es evidente que una nueva metodología docente requiere también de nuevos sistemas de evaluación. En este sentido, la evaluación inicial, a modo de diagnóstico, puede ser de utilidad, pero para docentes con muchos años de experiencia, no la veo imperativa. La propia práctica nos indica ya qué tipo de estudiantes tenemos y cómo suelen abordar el aprendizaje. Sí que me parece muy importante para que los propios estudiantes se pongan en lo que podemos denominar modo alerta, al detectar gracias a los cuestionarios iniciales su necesidad de apostar por la participación activa en un proceso de aprendizaje que es necesario para ellos, y además útil y relevante para 
su práctica profesional futura. Es decir, les permite tomar conciencia de que saben poco o nada sobre una cuestión que desearían poder responder. La evaluación y asignación de una calificación a cada alumno, según mi modelo ideal, debería ser continua, basada en el trabajo diario en el aula, y en el avance en el desarrollo del proyecto. Mi objetivo es eliminar completamente los exámenes, y evaluar en base a las distintas actividades de aprendizaje que se proponen durante el curso, la presencia activa en clase, y el resultado del proyecto finalmente desarrollado por los estudiantes. Para concluir, no quiero dejar de resaltar el papel que juega el entorno de aprendizaje, es decir, el horario, el aula, el número de estudiantes, el mobiliario y los equipos, que son parte fundamental de este modelo, difícil de aplicar en las aulas actuales de la Universidad de Sevilla, pensadas en su mayoría para un modelo pedagógico eminentemente transmisivo.

Jornadas de Formación e Innovación Docente del Profesorado | № 1 (2018) Esta obra se distribuye con la licencia Creative Commons 


\section{Referencias Bibliográficas}

Barrows, H.S. (1986). A Taxonomy of problem-based learning methods. Medical Education, 20(6), 481-486.

Benito, A.; Cruz, A. (2005). Nuevas claves para la docencia universitaria en el Espacio Europeo de Educación Superior. Madrid: Narcea.

De Miguel M. (coord.). (2005). Metodologías de enseñanza para el desarrollo de competencias. Orientaciones para el profesorado universitario ante el Espacio Europeo de Educación Superior. Madrid: Alianza.

Finkel, D. L. (2008). Dar clase con la boca cerrada. Valencia: Publicacions de la Universitat de València.

Maldonado Pérez, M. (2008). Aprendizaje basado en proyectos colaborativos. Una experiencia en educación superior. Laurus, Revista de Educación. 14(28), 150-180.

Marti, J.A; Heydrich, M.; Rojas, M.; Hernández, A. (2010). Aprendizaje basado en proyectos: una experiencia de innovación docente. Revista Universidad EAFIT, 4(158), $11-21$

Porlán, R. (Coord.) (2017). Enseñanza Universitaria. Cómo mejorarla. Madrid: Morata.

Prieto, L. (2006). Aprendizaje activo en el aula universitaria: el caso del aprendizaje basado en problemas. Miscelánea Comillas, Revista de Ciencias Humanas y Sociales, 64(124), 173-196.

Revilla, M.A.; López, J. (2010). El Aprendizaje Basado en Problemas en la Docencia del Marketing. En J.L. Jiménez-Caballero y A. Rodríguez Díaz (Coords.), Nuevas Enseñanzas de Grado en la EUEE de la Universidad de Sevilla. Granada: GEU.

Servicio de Innovación Educativa UPM (2008). Aprendizaje basado en problemas. Recuperado en https://innovacioneducativa.upm.es/guias/Aprendizaje_basado_en_ problemas.pdf

Jornadas de Formación e Innovación Docente del Profesorado | № 1 (2018) Esta obra se distribuye con la licencia Creative Commons 